\title{
El pensamiento y conocimiento del profesorado sobre la mediación para la inclusión de alumnado extranjero
}

\author{
Natalia Barranco Izquierdo - Universidad de Valladolid
}

0000-0002-7831-6158

Recepción: 25.06.2019 | Aceptado: 27.01.2020

Correspondencia a través de ORCID: Natalia Barranco Izquierdo

iD 0000-0002-7831-6158

Citar: Barranco Izquierdo, N (2020). El pensamiento y conocimiento del profesorado sobre la mediación para la inclusión de alumnado extranjero. REIDOCREA, 9, 63-72.

Resumen: En este artículo se presenta un estudio focalizado en el profesorado de inglés lengua extranjera de la Educación Primaria, como Agente mediador, a quien corresponde optimizar el proceso de inclusión del alumnado extranjero en las aulas. Partiendo del potencial de la mediación como actividad comunicativa de la lengua para la resolución de conflictos comunicativos y considerando que las actuaciones del profesorado están intrínsecamente relacionadas con su pensamiento y su conocimiento, nos planteamos indagar sobre este pensamiento y este conocimiento sobre la noción que nos ocupa de mediación en la perspectiva de anticipar elementos clave en la formación didáctica. Mediante la técnica de la encuesta, aplicamos un cuestionario a una muestra poblacional significativa de estos docentes y recogemos datos en torno a la noción actualizada de mediación lingüística, cultural, social y pedagógica (North y Piccardo, 2016), para dar cuenta de la relación significativa entre pensamiento, conocimiento y actuación.

Palabra clave: Pensamiento

\section{Teacher thinking and knowledge about mediation for the inclusion of foreign students}

Abstract: This paper presents a study focused on the English as a foreign language teacher in Primary Education. This teacher, who acts as a mediating agent, is in charge of optimizing the process of social and communicative integration of foreign students. Taking into account the potential of mediation as a communicative language activity for the resolution of communicative conflicts and considering that the actions of the teaching staff are intrinsically related to their thinking and knowledge, we aim to inquire about the thought and knowledge about the notion mediation in the perspective of anticipating key elements in didactic training. Through the survey technique, we applied a questionnaire to a significant sample of teachers and collected data on the updated notion of linguistic, cultural, social and pedagogical mediation (North and Piccardo, 2016), to give account on the significant relationship between thought, knowledge and action.

Keyword: Thinking

\section{Introducción}

Ante la confluencia en las aulas cada vez más generalizada de alumnado extranjero con variedades lingüísticas y diversidad cultural- desde los primeros niveles educativos, se plantean las problemáticas globales relativas a las relaciones pedagógicas. Problemáticas que focalizamos, en este caso, para el desarrollo de la materia de Inglés Lengua Extranjera [en adelante ILE], la cual forma parte de la competencia en comunicación lingüística y tiene como principales referentes en el currículo nacional las directrices y las pautas establecidas por el equipo de expertos de la División de Políticas Lingüísticas en el documento referencial Common European Framework of Reference for Languages: learning, teaching, assessment -en adelante, CEFR- (Council of Europe, 2001) y en su actualización (Council of Europe, 2018).

Para este alumnado extranjero escolarizado está contemplada su inclusión social y escolar -con un carácter bidireccional y dinámico de adaptación y como un ajuste mutuoen el proceso de su normalización escolar. Esta inclusión social y escolar es un reto en materia de calidad de la Educación, tal y como se recoge en el objetivo 4.5 "Gender equality and inclusion" de la Agenda 2030 para el Desarrollo Sostenible (United Nations, 
2015) y conlleva una evolución y un cambio en las responsabilidades y en las competencias que se espera configuren el perfil profesional del profesorado de ILE en los niveles de Educación Primaria (Kelly et al., 2004) y, en consecuencia, en los actos profesionales docentes que tienen lugar para y en el proceso de enseñanza-aprendizaje. En este orden de cosas, aludimos a los aspectos propios del funcionamiento escolar, como a los aspectos de la enseñanza-aprendizaje de ILE que requieren de intervención didáctica en el seno del contexto escolar conforme a los distintos niveles de organización curricular (Van den Akker et al., 2006).

Para el logro, pues, de esta inclusión -en la doble dimensión que compete a los docentes en las aulas, esto es, la que subyace a las relaciones que tienen lugar en la situación pedagógica contextual (social) y la de la comunicación en lengua extranjera como competencia básica curricular (escolar)-, la mediación es genéricamente valorada como un metanivel organizador de las relaciones entre los individuos. Se nos presenta como un concepto polisémico, por cuanto que: a) se entiende en cualquier ámbito de acción social, como un instrumento de gestión de transacciones (Bondu, 1998); b) se traspone a las relaciones sociales entre los participantes en la situación pedagógica mediante la que se establece un sistema de regulación (Lenoir, 2008) que utiliza los intercambios discursivos en dos dimensiones interdependientes, a saber: la lingüística y la cultural/ intercultural; c) se nos muestra socio-educativamente, en la relación de enseñanzaaprendizaje de cada situación de enseñanza-aprendizaje concreta, como un instrumento a través de medios verbales y no verbales; d) se convierte en el aula de ILE -como lugar social institucional de referencia-, en una competencia en sí misma, por cuanto que vertebra las relaciones pedagógicas en el desarrollo del proceso de enseñanza-aprendizaje, para resolver los conflictos comunicativos entre los participantes en los intercambios discursivos en torno al Objeto de conocimiento que es el ILE. Tales conflictos comunicativos dificultan el acceso a los contenidos curriculares generando conflictos cognitivos y, por lo tanto, la normalización de este alumnado en las aulas de referencia, tal y como está establecido en Ley Orgánica 8/2013, de 9 de diciembre, para la mejora de la calidad educativa -LOMCE.

Esta polisemicidad es recogida por North y Piccardo (2016) quienes enumeran cuatro tipos fundamentales de mediación y ofrecen, por primera vez, descriptores ilustrados para esta actividad. Estos autores distinguen entre mediación lingüística, que alude a las lenguas que intervienen en la interacción (Corcoll López y González-Davies, 2016); mediación cultural, que focaliza en la conciencia cultural/intercultural (Barret, 2013; Byram, 2009); mediación social, que se vincula a la anticipación y resolución de conflictos mediante la gestión de las interacciones (Coste y Cavalli, 2015); y mediación pedagógica (Delgado Álvarez, 2013), que se refiere a la mediación cognitiva y a la mediación relacional.

La mediación se nos muestra con un estatus particular, potencial y efectivo, que afecta a toda situación de enseñanza-aprendizaje. De forma particular, en el ámbito disciplinar de Didáctica de la Lengua y la Literatura se establece como una actividad comunicativa de la lengua que, en términos de competencia, se aborda como un conjunto de conocimientos, capacidades y actitudes. La atención a la mediación como tal actividad comunicativa de la lengua no contemplada anteriormente en el currículo de lengua extranjera, o al menos no de forma explícita, en gran medida puede ser considerada como uno de los aspectos evolutivos en los trabajos de la División de Políticas Lingüísticas del Consejo de Europa (CEFR, 2001, 2018).

La definición que de ella encontramos en estos documentos referenciales demanda nuestra atención para el desarrollo curricular de la competencia clave 2 "Comunicación en lenguas extranjeras" (Diario Oficial de la Unión Europea, 2006), por cuanto que 
desvela sus potencialidades y virtualidades en términos de existencia y funcionalidad. Las actividades de mediación, escritas y orales, hacen posible la comunicación entre personas que son incapaces, por cualquier motivo, de comunicarse entre sí directamente, por lo que poseen un lugar importante en el funcionamiento normal de nuestras sociedades.

La mediación ocupa, por lo tanto, un lugar central como eje vertebrador de la comunicación, por cuanto que, en esta actividad comunicativa de la lengua, tal y como se refiere en el CEFR (2001) el usuario de la lengua se preocupa simplemente de actuar como intermediario entre personas que no se pueden entender por tener, principal, aunque no exclusivamente, una lengua primera diferente. Si bien, aunque la atención prestada en este documento a la mediación como actividad comunicativa de la lengua es pionera, podemos afirmar que es limitada. En la actualización de este documento se matiza sobre los diferentes contextos en los que se pone en funcionamiento, a saber:

In mediation, the user/learner acts as a social agent who creates bridges and helps to construct or convey meaning $[. .$.$] creating the space and conditions for communicating$ and/or learning, collaborating to construct new meaning, and passing on new information in an appropriate form. The context can be social, pedagogic, cultural, linguistic or professional (CEFR, 2018, p.103).

Para el ámbito que nos ocupa, se atiende a las condiciones formales de la existencia de la mediación y su desarrollo, en los siguientes términos "El mediador por excelencia sigue siendo el profesor, cuya mediación consiste en interactuar con el alumno en su zona de aprendizaje, usando una variedad de intercambios discursivos, como ejemplificaciones de estrategias de aprendizaje e impulsos para realizar nuevas tareas [...]". (Arroyo González, 2004, p. 224-225)

Su trasposición a la situación de enseñanza-aprendizaje de lenguas extranjeras, nos ha llevado a evidenciar: a) su carácter pluridimensional, en el sentido de que concierne tanto a la enseñanza como al aprendizaje de lenguas y se aborda al mismo tiempo como objeto de enseñanza y de aprendizaje; b) su carácter plurimodal, ya que puede considerarse como objeto y, sobre todo, como instrumento, por cuanto que para su movilización se puede usar la lengua oral o la lengua escrita o ambas simultáneamente, etc.; c) su carácter multicomponencial, ya que varias de las otras actividades comunicativas de la lengua -expresión, comprensión e interacción- pueden estar implicadas en su movilización.

Es a cada profesor -Agente, recurso humano en las relaciones pedagógicas- y encargado de la competencia clave 2, a quien corresponde, en última instancia, resolver las problemáticas derivadas de la diversidad de alumnado en las aulas, en particular, para el caso que nos ocupa, aulas de ILE. Ante estas consideraciones, exponemos las siguientes premisas:

- Con la presencia de alumnado extranjero en las aulas, es objeto de atención la ruptura del vínculo de comunicación durante el proceso de enseñanzaaprendizaje de ILE. Esta ruptura entronca, teórica y conceptualmente, con la noción consolidada de conflicto comunicativo.

- En cada situación de enseñanza-aprendizaje, la mediación vertebra los fenómenos comunicativos que tienen lugar en las aulas de ILE con alumnado extranjero.

- La mediación, por su carácter pluridimensional, plurimodal o pluri-instrumental y multicomponencial, presenta potencialidades y virtualidades para la resolución 
de los conflictos comunicativos producidos en el aula de ILE con alumnado extranjero.

- La toma de conciencia por parte del profesorado de ILE del alcance y los efectos de la mediación en la perspectiva de la inclusión social y escolar, mediante su intervención didáctica, permitirá atender a la resolución de los conflictos comunicativos. Esto hará preciso que la mediación se contemple como parte integrante competencial $y$, por tanto, objeto de la formación didáctica de este profesorado, revelándose como configuradora de su perfil profesional. Para la Didáctica de las Lenguas-Culturas y, en concreto, para el área de conocimiento de Didáctica de la Lengua y la Literatura, la mediación se establece como una actividad comunicativa de la lengua que, en términos de competencia, se aborda como un conjunto de conocimientos (saber o conocimiento declarativo), capacidades (saber-hacer instrumental) y actitudes (saber-ser intencional).

El acto profesional de conocer -asegurado por la formación tanto inicial como continuapreside y es necesario para la realización de cada uno de los otros actos profesionales de tomar decisiones, aplicar y evaluar, cuya concreción corresponde a la planificación y a la intervención (Guillén Díaz y Castro Prieto, 1998). Es decir que el conocimiento y pensamiento del profesorado sobre las potencialidades de la actividad comunicativa de la lengua denominada mediación para la resolución de los conflictos comunicativos, tiene efectos directos en sus actuaciones durante el proceso de enseñanza-aprendizaje, por lo que su consideración es fundamental en la perspectiva última de su formación didáctica.

\section{Objetivo}

El objetivo general de este artículo es acercarnos a las percepciones -en términos de pensamiento y conocimiento- del profesorado de ILE en Educación Primaria con alumnado extranjero en sus aulas, sobre la noción de mediación.

La relación significativa entre pensamiento-conocimiento-actuación nos permite aventurar potenciales actuaciones de formación respecto a un conocimiento declarativo, un saber-hacer instrumental y un saber-ser intencional que focalicen en esta actividad comunicativa de la lengua.

\section{Método}

\section{Participantes, instrumento y procedimiento}

Para constatar la presencia de alumnado extranjero en las aulas accedimos a datos facilitados por la Junta de Castilla y León, en concreto por el área de Atención a la Diversidad de la Consejería de Educación. La información facilitada, no disponible por ningún otro medio y con carácter confidencial, nos ofrecía datos de contexto relativos al alumnado extranjero escolarizado en las nueve provincias de la Comunidad.

Distribuimos a profesorado de ILE -de Centros públicos y concertados de Educación Primaria de la Comunidad Autónoma de Castilla y León con alumnado extranjero en sus aulas-, 179 cuestionarios de los que nos fueron devueltos 66 (43 de centros públicos y 23 de centros concertados). Los datos se han tratado de forma manual mediante la técnica de análisis de contenido.

La concepción y elaboración del cuestionario se ha llevado a cabo en función de cuatro ejes de datos estimados necesarios y significativos: 
- $1^{\circ}$ eje: tres ítems (del 1 al 3) para dar cuenta de su saber y su saber-ser ante la presencia de alumnado extranjero en las aulas, haciendo uso- en los dos últimos ítems- de la escala de tipo Likert, con cinco niveles de respuesta.

- $2^{\circ}$ eje: tres ítems (del 4 al 6) para dar cuenta de su saber-hacer en los actos profesionales de planificación e intervención. En concreto, el ítem 6 da cuenta de un saber-hacer instrumental.

- 3er eje: cinco ítems (del 7 al 11) para dar cuenta de su saber relativo a la noción mediación como un conocimiento declarativo en sí mismo y cómo saber efectivo para y en el aula, es decir, de orden organizativo curricular escolar.

- $4^{\circ}$ eje: dos ítems (el 12 y el 13) para dar cuenta de su saber-hacer focalizados en los conflictos comunicativos en el aula de ILE con alumnado extranjero.

Se completa con un ítem (sin numerar) relativo a la propia experiencia del profesorado en la resolución de un conflicto comunicativo mediante la mediación.

\section{Análisis de los datos e interpretación de los resultados}

Los datos de cada uno de los ítems del cuestionario han sido explorados e interpretados de forma univariable. Para las respuestas se usó la escala Likert con la siguientes posibilidades: nada, un poco, algo, bastante, mucho/muy.

\section{$1^{\text {er }}$ eje de datos}

Respecto al ítem 1, relativo a la consideración del profesorado de ILE sobre la preparación para atender la presencia de alumnado extranjero en las aulas, los datos muestran que el $67 \%$ de los encuestados manifiesta que el profesorado en general está "nada" preparado para atender la presencia de alumnado extranjero. Esto pone de relieve su desconocimiento para dar una respuesta efectiva a las necesidades específicas que este alumnado pueda presentar, asociadas a situaciones sociales 0 culturales desfavorecidas y marcadas por dificultades de acceso, permanencia y/o promoción en el sistema educativo.

Respecto al ítem 2, relativo a la creencia del profesorado de ILE sobre cómo afecta la presencia de alumnado extranjero en el aula, los datos revelan que este profesorado, a pesar de haber manifestado en el ítem 1 que considera no estar preparado para atender la presencia de alumnado extranjero en las aulas, muestra una actitud muy positiva hacia la misma, ya que un $84 \%$ afirma que este alumnado contribuye "bastante" o "mucho" al enriquecimiento de las clases. Se revela su toma de conciencia sobre esta presencia afectando a las programaciones, al desarrollo de la clase y a la generación de conflictos lingüísticos y culturales. Es destacable el hecho de que un $39 \%$ manifiesta que este alumnado genera conflictos personales, lo cual afecta de manera directa a la inclusión social.

Respecto al ítem 3, relativo a la consideración del profesorado sobre lo que estima necesario en la perspectiva de atender la presencia de alumnado extranjero en las aulas, los datos muestran que el $70 \%$ del profesorado considera la formación específica "bastante" o "muy necesaria", en la perspectiva de atender las necesidades específicas del alumnado extranjero en las aulas. Al mismo tiempo, se observa que el $36 \%$ señala la presencia de mediadores culturales en términos de "bastante" o "algo" necesarios. Cabría incidir en que el papel de los mediadores culturales, según las Administraciones educativas, consiste en reducir lo máximo posible las diferencias culturales de los alumnos de llegada y los de acogida, esto es, en evitar en la mayor medida posible los conflictos personales. Este dato se podría prever si tenemos en cuenta que un $39 \%$ ha manifestado que este alumnado genera este tipo de conflictos. 


\section{$2^{\circ}$ eje de datos}

Respecto al ítem 4 y al ítem 5 , relativos a la planificación de la clase en torno a la gestión de problemas y al logro de metas, presentamos los datos en el Gráfico 1 y en el Gráfico 2 respectivamente.

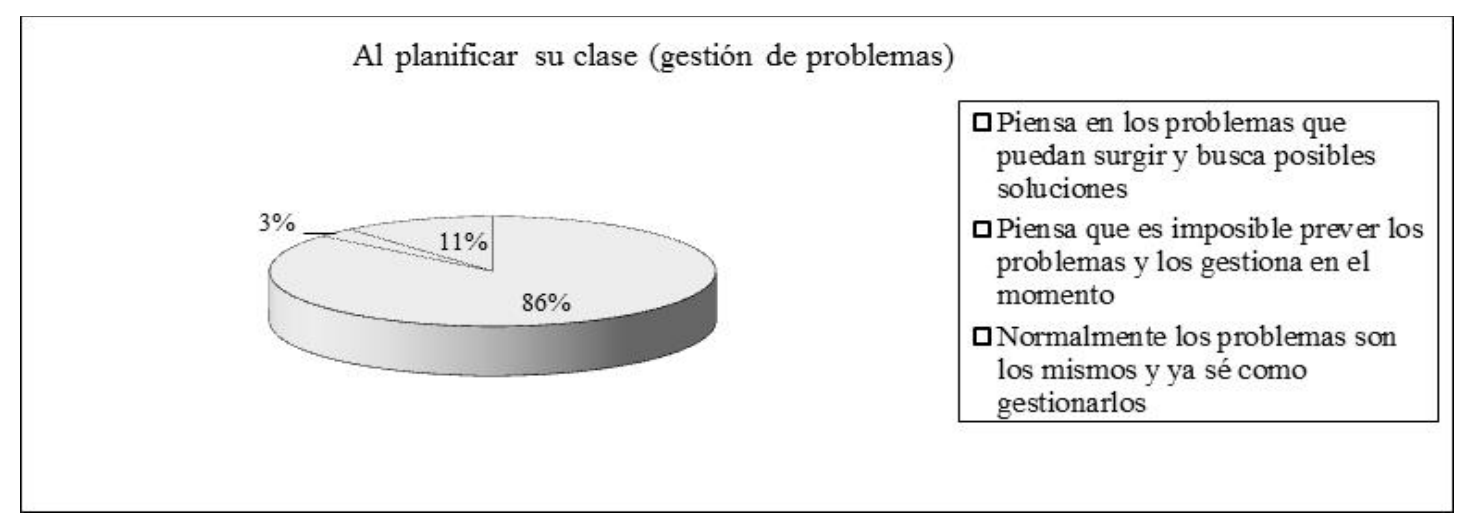

Gráfico 1. Datos relativos a la planificación de la clase: la gestión de problemas.

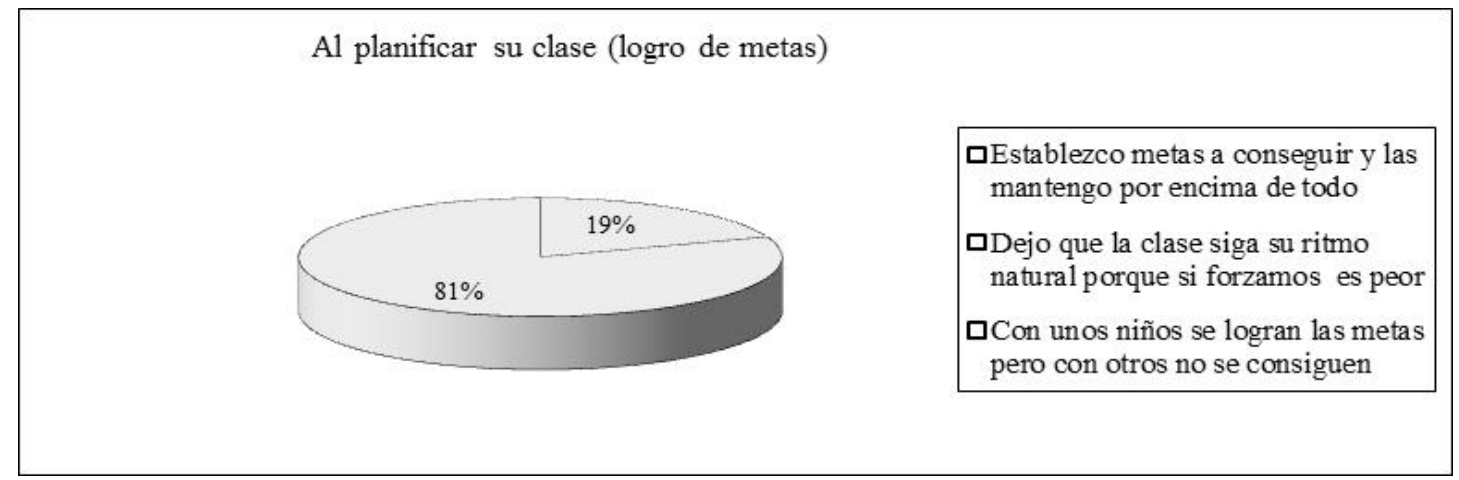

Gráfico 2. Datos relativos a la planificación de la clase: el logro de metas.

En el momento de planificar las clases, un $86 \%$ del profesorado afirma que piensa "algo" o "bastante" en posibles soluciones a los potenciales problemas que puedan surgir. Si bien, paradójicamente, el $84 \%$ sostiene que las metas que se establecen al iniciar el curso no son alcanzadas por todo el alumnado, asumiendo que las posibles soluciones planificadas a los potenciales problemas no van a ser efectivas y eficientes.

En el ítem 6 se pide al profesorado que otorgue, de forma numérica (de 1 a 10, de poca importancia a mucha importancia), la importancia a los siguientes enunciados:

- Relacionar la enseñanza de los alumnos con lo que ya saben

- Fomentar la curiosidad intelectual en sus alumnos

- Fomentar su sentimiento de capacidad de lograr metas

- Favorecer que los alumnos sepan que hacer, cómo, cuándo y porqué

- Fomentar el interés y respeto por el grupo

- Fomentar actitudes de cooperación con el grupo

- Potenciar la discusión reflexiva

- Atender a las diferencias individuales de cada alumno

- Desarrollar en ellos actitudes positivas

- Fomentar el respeto de la individualidad y de sus propios ritmos de aprendizaje

- Resolver de conflictos de comunicación 
- Aprovechar las diferencias para enriquecer a los alumnos

- Fomentar el diálogo como solución de conflictos de comunicación

Los datos no aportan información distintiva ya que prácticamente la totalidad de los encuestados puntúan con 9 ó 10 a cada uno de los enunciados. En todo caso, cabría destacar que las puntuaciones más bajas se refieren a:

- Potenciar la discusión reflexiva.

- Fomentar el diálogo como solución de conflictos de comunicación.

Es un dato revelador el hecho de que los enunciados que hacen referencia a la discusión reflexiva y al diálogo sean los menos valorados. Esto demuestra, en cierto modo, que no se contemplan como prioridades en la enseñanza-aprendizaje en aulas de ILE en Educación Primaria, mientras que, desde las recomendaciones europeas, este tipo de participación activa se concibe como uno de los pilares en materia de Educación. Asimismo, desde los fundamentos de la mediación, se mantiene que ésta contribuye al desarrollo de la capacidad de diálogo y de discusión reflexiva, capacidad que, sin duda, conduce a la resolución de los conflictos comunicativos. Esto es, una menor presencia de discusión reflexiva y de diálogo en las aulas, comportaría, lamentablemente, una menor presencia de la mediación.

\section{$3^{\text {er }}$ eje de datos}

Respecto al ítem 7, relativo al nivel de conocimiento del profesorado sobre las actividades comunicativas de la lengua del $\operatorname{CEFR}(2001,2018)$, los datos muestran que un $92 \%$ del profesorado afirma tener "nada" o algo" de conocimiento sobre las actividades comunicativas de la lengua, lo que incluye a la mediación que nos ocupa.

Respecto al ítem 8, relativo al nivel de conocimiento del profesorado sobre la mediación, en concreto, como actividad comunicativa de la lengua, los datos constatan que a pesar de que un $92 \%$ declara tener "nada" o algo" de conocimiento sobre las actividades comunicativas de la lengua, el 33\% afirma que la mediación sí es una de éstas, lo cual es difícilmente interpretable de forma conjunta. Al mismo tiempo, un $48 \%$ afirma que la mediación sí es una actividad comunicativa de la lengua pero no al mismo nivel que las otras, reforzándose así la eventual interpretación de los datos obtenidos del ítem 7.

Respecto al ítem 9, relativo la utilización o a la percepción de su utilización de la mediación en las clases, los datos revelan que un $77 \%$ del profesorado está "muy" seguro de que sí que utiliza la mediación en sus clases. De nuevo, este alto porcentaje se puede deber al hecho de que el término mediación sea de uso generalizado en los ámbitos educativos para la resolución de conflictos de tipo personal, es decir, que se la vincula a la vertiente social de su significado. En este sentido, las siguientes respuestas nos confirman este grado de familiaridad.

Respecto al ítem 10, relativo a la consideración del profesorado sobre el interés que tiene la mediación para o con el alumnado extranjero, presentamos los datos en el Gráfico 3. 
La mediación tiene especial interés para alumnos extranjeros

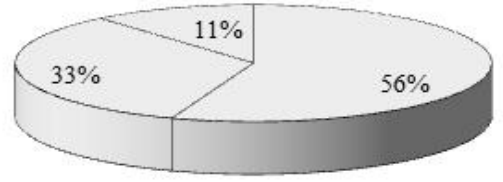

口Claro, les ayuda a superar conflictos de comunicación

$\square$ Tiene el mismo interés para todos los alumnos

$\square$ Al contrario, tiene más interés para el resto de los alumnos

№ lo sé

Gráfico 3. Datos relativos a la consideración del profesorado sobre el interés que tiene la mediación para/con el alumnado extranjero.

Un $56 \%$ manifiesta que la mediación tiene un interés especial para el alumnado extranjero por cuanto que le ayuda a superar conflictos de comunicación, siendo consciente de las potencialidades y virtualidades que tiene la mediación para este alumnado en particular.

Respecto al ítem 11, relativo a las percepciones del profesorado sobre quién o qué puede ejercer la función de Agente mediador en el aula, presentamos los datos en el Gráfico 4.

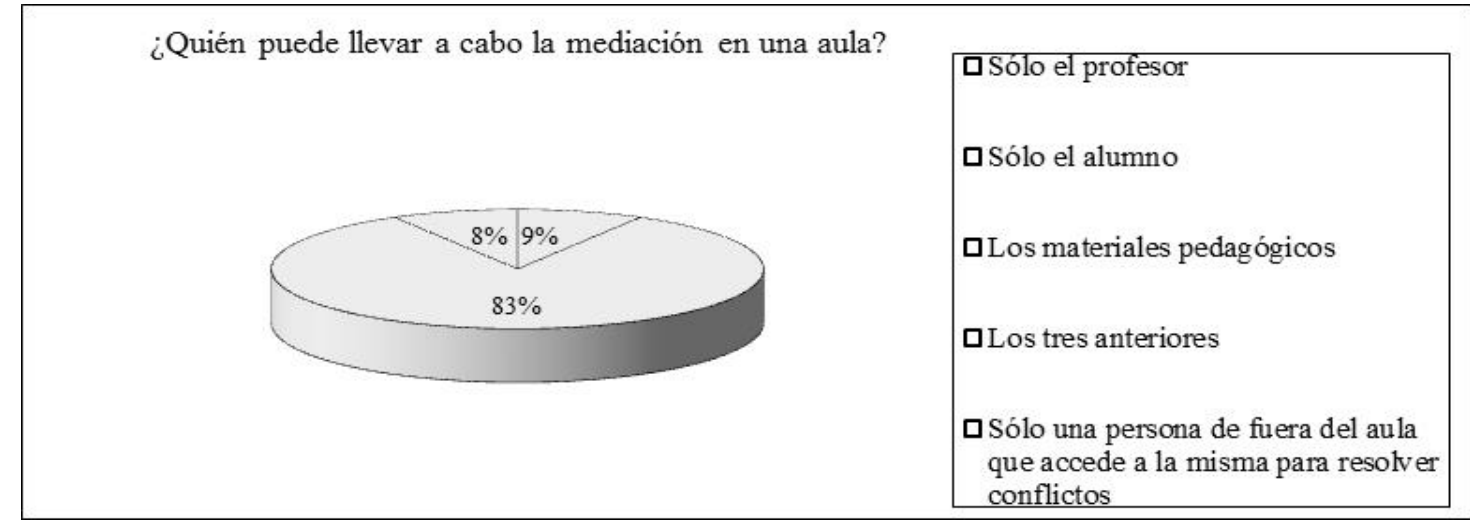

Gráfico 4. Datos relativos a las percepciones del profesorado sobre quién o qué puede ejercer la función de Agente mediador en el aula.

El $84 \%$ del profesorado afirma que tanto el profesor, como el alumno, como los materiales pedagógicos pueden ser mediadores en un aula. Lo más relevante de estas respuestas es que el $8 \%$ sostiene que solamente una persona externa al aula puede ser mediador y ayudar a resolver conflictos. En este caso, la mediación es exclusivamente vinculada a la resolución de conflictos personales y asociada a la presencia de un mediador cultural.

\section{$4^{\circ}$ eje de datos}

Respecto al ítem 12 y al ítem 13, relativos a las percepciones del profesorado de ILE encuestado sobre los problemas que puede generar el hecho de poseer una lengua o una cultura primera diferente para el proceso de la comunicación (aludiendo a la mediación intra- o intralingüística), los datos obtenidos son significativos, ya que un 19\% del profesorado considera, imprevisiblemente, que el hecho de que un alumno tenga una cultura primera diferente en un aula de ILE puede suponer un problema "enorme" 
para la comunicación, mientras que en ningún caso se ha considerado del mismo modo el hecho de que un alumno tenga una lengua primera diferente.

Respecto al último ítem del cuestionario, en el que -apelando a la propia experiencia del profesorado- se instaba a narrar un problema de comunicación acaecido en el aula y para cuya resolución se haya empleado la mediación (se ha usado de manera intencional el término específico), obtuvimos narraciones en las que podemos identificar los elementos de la movilización de la mediación en sus aspectos teóricos y metodológicos subyacentes.

En los ocho cuestionarios en los que encontramos completado este ítem, lo más destacable en cuanto a los aspectos teóricos es que en uno de los casos el profesor manifiesta que no puede dar una respuesta por desconocimiento del término; en cuanto a los aspectos metodológicos subyacentes al término en cuatro casos los profesores afirman que para solventar problemas de comunicación en el aula han recurrido tanto a la comunicación no verbal a través de múltiples acciones paralingüísticas y/o elementos paratextuales, tales como: el lenguaje corporal, las fotografías o los dibujos; como a la comunicación verbal a través de estrategias de ejecución, tales como: poner ejemplos; en otros dos casos, los profesores afirman que los alumnos tienen una implicación activa ya que se ayudan unos a otros a resolver los problemas de comunicación, ejerciendo ellos mismos de Agentes mediadores; y finalmente, en un caso, la profesora manifiesta que recurre al establecimiento de vínculos afectivos que aluden a la autoestima, la implicación, la motivación, el estado y la actitud del alumnado, los cuales tienen sus efectos, a su vez, en la eficacia y en la eficiencia de los aprendizajes.

\section{Discusión}

El análisis de las informaciones obtenidas como percepciones del profesorado de ILE encuestado en torno a la noción de mediación, y la interpretación de los resultados, nos llevan a considerar como elementos clave en la formación didáctica del profesorado de ILE de Educación Primaria tres órdenes de saberes, contemplados en la perspectiva competencial del perfil profesional docente de este profesorado: un saber o conocimiento declarativo sobre esta noción en su carácter pluridimensional, plurimodal y multicomponencial, y vertebrador de la resolución de conflictos comunicativos; un saber-hacer instrumental para/en la intervención en el aula, y un saber-ser intencional de disposición positiva. La posesión de estos tres saberes otorga, previsiblemente, al profesorado de ILE unas mejores condiciones para favorecer la inclusión del alumnado extranjero en sus aulas.

Un saber o conocimiento declarativo, en el sentido de sensibilizar al profesorado sobre las potencialidades efectivas de la mediación, procedentes de su alcance y de sus efectos en la resolución de los conflictos comunicativos y cognitivos subyacentes que tienen presencia en el aula con alumnado extranjero, lo cual se ve sustentado por cuanto que:

- Un 92\% afirma tener un conocimiento prácticamente nulo sobre las actividades comunicativas de la lengua, y consecuentemente, sobre sus potencialidades.

- El 36\% señala como "muy" o "bastante" necesaria la presencia de mediadores culturales para atender al alumnado extranjero, asociándolos, erróneamente, con los conflictos personales.

- El $48 \%$ afirma que la mediación es una actividad comunicativa de la lengua, pero no al mismo nivel que las otras. 
Un saber-hacer instrumental, en el sentido de estar en las mejores condiciones de optimizar la movilización de la actividad comunicativa de la lengua de mediación de forma intencional en sí misma, o a través de las otras actividades comunicativas de la lengua, para la resolución de los conflictos comunicativos y cognitivos subyacentes, por cuanto que:

- El $67 \%$ manifiesta explícitamente su desconocimiento a cómo dar una respuesta efectiva a las necesidades específicas de este alumnado.

- El $34 \%$ otorga puntuaciones muy bajas a los enunciados relacionados con la potenciación y el fomento de la discusión reflexiva y del diálogo, siendo éstos, elementos fundamentales para el desarrollo de la actividad comunicativa de la lengua de mediación.

En cuanto a un saber-ser intencional, en el sentido de estar dispuesto de forma positiva y actuar en consecuencia ante la presencia de heterogeneidad en el aula, cabría considerar que es un componente competencial que no necesitaría prever una formación didáctica, por cuanto que:

- El $84 \%$ del profesorado encuestado afirma que el alumnado extranjero contribuye "mucho" o "bastante" al enriquecimiento de las clases, mostrando una predisposición manifiesta hacia la presencia de este alumnado en las aulas.

\section{Referencias}

Arroyo González, R (2004). "Mediación didáctica". En F Salvador Mata, JL Rodríguez Diéguez y A Bolívar Botía (Dirs.), Diccionario Enciclopédico de Didáctica, II (224-225). Málaga: Aljibe.

Barrett, M (2013). Intercultural competence: A distinctive hallmark of interculturalism? In M. Barrett (Ed.), Interculturalism and multiculturalism: Similarities and differences (147-168). Strasbourg: Council of Europe Publishing.

Bondu, D (1998). Nouvelles pratiques de médiation sociale. Paris: ESF.

Byram, M (2009). The intercultural speaker and the pedagogy of foreign language education. In DK Deardorff (Ed.), The Sage handbook of intercultural competence (321-332). Los Angeles CA: Sage.

Corcoll López, C y González-Davies, M (2016). Switching codes in the plurilingual classroom. ELT Journal, 70, 67-77.

Council of Europe (2001). Common European Framework of Reference for Languages: learning, teaching, assessment. Strasbourg: Modern Languages Division.

Council of Europe (2018). Common European Framework of Reference for Languages: learning, teaching, assessment. Companion volume with new descriptors. Strasbourg: Education Policy Division.

Coste, D, y Cavalli, M (2015). Education, mobility, otherness. The mediation functions at schools. Strasbourg: Council of Europe.

Delgado Álvarez, A (2013). Pedagogical mediation and learning. Revista de Lenguas Modernas, 19, 513-522.
Diario Oficial de la Unión Europea (2006). Recomendación del Parlamento Europeo y del Consejo, de 18 de diciembre de 2006, sobre las competencias clave para el aprendizaje permanente (2006/962/CE). L 394 de 30.12.2006.

Guillén Díaz, C y Castro Prieto, P (1998). Manual de autoformación para una didáctica de la lengua-cultura extranjera. Madrid: La Muralla.

Kelly, M, et al. (2004). European Profile for Language Teacher Education. A Frame of Reference. Southampton: Universidad de Southampton.

Lenoir, Y (2008). Les finalités en éducation: un cadre conceptuel pour cerner les positionnements épistémologiques et axiologiques. En D. Favre, A. Hasni y C. Reynaud (Dirs.), Les valeurs explicites et implicites dans la formation des enseignants. Entre "toujours plus" et "mieux vivre ensemble" (39-53). Bruxelles: De Boeck.

Ley Orgánica 8/2013, de 9 de diciembre, para la mejora de la calidad educativa (LOMCE). BOE 295 del 10.12.2013.

North, B, \& Piccardo, E (2016). Common European Framework of Reference for Languages: learning, teaching, assessment. Developing illustrative descriptors of aspects of mediation for the CEFR. Strasbourg: Council of Europe.

United Nations (2015). Transforming our world: the 2030 Agenda for Sustainable Development. New York: United Nations.

Van den Akker, J, et al. (Eds.) (2006). Educational design research. London: Routledge. 